\title{
Pedagogía Cognitiva: La educación y el estudio de la mente en la Sociedad de la Información
}

\author{
Gonzalo Vázquez Gómez \\ Fernando Bárcena Orbe \\ Universidad Complutense de Madrid \\ (España)
}

\section{Introducción: educación y cultura en la sociedad cognitiva}

Hace algunos años, en un trabajo sobre la pedagogía cognitiva, se partía de este postulado básico:[1]

la sociedad actual se caracteriza por la generación, desarrollo y difusión del conocimiento. Los cambios humanos son de tal calidad y calibre que han provocado una verdadera revolución científica y social sólo comparable -para algunos, incluso, superior- a las previas revoluciones industriales. Si cada estado de cosas requiere un tipo de pedagogía, una escuela, ¿qué tipo de pedagogía requieren los tiempos actuales?

Esta pregunta tiene diversos significados. Primero, que cada sociedad implica un tipo de educación, instituye (crea) un tipo de escuela y alienta una forma de pensar la educación[2]. Segundo, que el progreso de la ciencia produce cambios en la estructura del pensamiento [3]. Tercero, que la nueva sociedad, y la evolución de la ciencia y de la tecnología como construcciones sociales, requiere una nueva pedagogía.

En la base de este postulado y de esta pregunta subyace la creencia de que el complejo, y sin duda largo en el tiempo, proceso de aprendizaje humano responde no sólo a una especie de diseño genético, sino de modo principal y aún más significativo a un diseño cultural. A un diseño que toma cuerpo en el cerebro humano, máximo modelo y exponente de un sistema complejo [4].

En efecto, el aprender no expresa únicamente un mecanismo o propiedad adaptativa al entorno, función que los humanos compartimos con otros animales, sino que, sobre todo, es una propiedad atributiva. Por el aprender, y por lo que aprendemos -cuando este aprendizaje es verdaderamente humano- logramos incrementar nuestra competencia como hábiles mentalistas, seres capaces de atribuir, tanto a nosotros mismos como a los demás, diversos estados mentales como base de nuestra conducta y acción. Ello hace posible no sólo la comunicación con los demás, sino mejorar nuestra habilidad para elaborar sentido y descubrir significado al mundo.

A través del aprendizaje nos incorporamos, pues, a la cultura humana, incorporamos esa cultura de modo personal y generamos, además, nuevas formas de aprendizaje humano. De este modo, cada sociedad y cada cultura es creada por los procesos humanos de aprendizaje y, a su vez, genera nuevas formas de aprendizaje, es decir, una verdadera cultura de aprendizaje. Así, que lo que desde un primer análisis podría juzgarse sólo en términos de un mero aprendizaje de la cultura, acaba conduciendo a una auténtica, y en cierto modo nueva, cultura del aprender.

Esta cultura del aprendizaje se encuentra hoy propiciada y condicionada por los rasgos 
pedagógicos más llamativos de nuestras complejas sociedades modernas. La peculiaridad de esta sociedad es que la energía sobre la que se opera es la información, una energía, por así decirlo, inmaterial, distinta y más avanzada que las energías (el carbón, la electricidad) sobre las que se desarrollaron las primeras revoluciones industriales[5]. Dentro del sistema educativo cada individuo es un generador de energía y las políticas de reforma educativa se asientan en el principio de que cada individuo debe estar capacitado para diseñar su propio proceso de cualificación y para establecer relaciones cognitivas con un entorno (no sólo externo, sino también interno en el sentido que Luhman presta al enfoque de que los sistemas incluyen las relaciones con su entorno y al entorno mismo [6]), a un entorno cada vez más complejo.

Nuestras sociedades, así, son sociedades de la información, sociedades del aprendizaje, sociedades del conocimiento y sociedades educativas. Se genera un potencial educativo basado en diversos principios, a saber: el incremento de la plasticidad cerebral, la prolongación del período de formación a lo largo de toda la vida, el incremento de la demanda de formación, la diversificación de nuevos itinerarios formativos que propicia un flujo mayor de información, el desarrollo de las nuevas tecnologías de la información y la distribución del conocimiento a nuevas instituciones y centros de formación. Dentro de la sociedad cognitiva, las organizaciones con capacidad de adaptación dinámica, de proyección y supervivencia son, precisamente, las organizaciones cognitivas [7] . En este sentido, las tecnologías cognitivas, como formas de pensar, incluyen y superan a la propia tecnología .

\section{Hacia una Pedagogía cognitiva.}

La Pedagogía cognitiva, como ámbito de estudio, investigación y punto de apoyo para la toma de decisiones educativas, en contextos tanto formales como no formales, toma asiento precisamente en la necesidad de responder a esta demanda de aprendizaje a lo largo de toda la vida, de información y de conocimiento distribuido. Es decir, encuentra una primera justificación como consecuencia de los rasgos que actualmente toma la educación en nuestras sociedades cognitivas: en pocas palabras, la Pedagogía cognitiva es la pedagogía de la sociedad cognitiva.

En este sentido, aunque este campo de estudio e investigación está todavía por perfilar y asentarse, a la Pedagogía cognitiva le interesa, de una parte, el análisis de las dimensiones cognitivas de la educación en el marco de nuestras sociedades del conocimiento, y de otro lado, el estudio pedagógico de los procesos de pensamiento dentro del marco de las nuevas teorías de la mente, cuyos modelos y paradigmas parecen estar cambiando, al haberse operado un desplazamiento de interés desde el modelo del procesamiento de información a modelos psicoculturales de construcción de significado.

Interesa en un primer momento discernir entre cognición y pensamiento. A juicio de Arendt [8], ha de partirse de la aceptación de que pensamiento y cognición no son una misma cosa pues, mientras que el primero es el origen de las obras de arte y se manifiesta en la filosofía sin modificación alguna, la segunda persigue un objetivo movido por consideraciones prácticas y, una vez alcanzado, ahí finaliza. La cognición es un proceso útil, con principio y fin. Se trata de un proceso artificial y, como actividad, una actividad productiva. De acuerdo con esta forma de pensar, si la educación opera a través de los procesos cognitivos, a lo más que puede aspirar es a la construcción de artefactos. ¿Es, pues, toda inteligencia humana una inteligencia artificial y el propio cerebro humano (el cerebro con representaciones mentales contenidas en él y que lo modifican y originan continuamente) una construcción artificial? ¿Es la Pedagogía cognitiva una pedagogía de la construcción artificial del hombre? ¿Es un artefacto cultural el hombre educado? [9]. 
Nos interesa saber cómo se estructura la mente humana, y responder a la pregunta "¿qué es pensar?", como punto de partida para intervenir pedagógicamente en la mente dentro de contextos pedagógicamente configurados con mayor o menor grado de formalidad Y, por lo mismo, nos importa también el estudio de las dimensiones cognitivas, mentalistas y reflexivas vinculadas al proceso de intervención o de acción educativa y pedagógica.

Este tipo de intereses ha sido recientemente expuesto por Bruner (1997) quien aboga por una comprensión de la mente humana que se sitúe más allá de las teorías hoy dominantes en psicología cognitiva; lo que dice debería hacernos pensar:

la actividad mental humana no se conduce en solitario ni sin asistencia, incluso cuando sucede "dentro de la cabeza". Somos la única especie que enseña de una forma significativa. La vida mental se vive con otros, toma forma para ser comunicada, y se desarrolla con la ayuda de códigos culturales, tradiciones y cosas por el estilo. Pero esto va más allá de la escuela. La educación no sólo ocurre en las clases, sino también alrededor de la mesa de comedor (...), cuando los chicos intentan ayudarse unos a otros a dar sentido al mundo adulto, o cuando un maestro y un aprendiz interactúan en el trabajo. De manera que no hay nada más apropiado que la práctica educativa para probar una psicología cultural (p. 13).

La mente y los procesos cognitivos que incluye no están destinados únicamente a "procesar información" o a "resolver problemas", sino a dar sentido al mundo y a nosotros mismos, a través del redescubrimiento constante, con los demás y en espacios culturales, de nuevos sentidos y del significado.

¿Puede llegar la Pedagogía a familiarizarse con la "revolución cognitiva", a entrar dentro de la familia de las ciencias y las tecnologías cognitivas? Según dice Bruner (1991) el principal objetivo de la Revolución cognitiva fue recuperar el estudio de la mente en las ciencias humanas tras un período largo de fuerte objetivismo. La Revolución cognitiva da origen así a la Psicología cognitiva, cuyo nacimiento es paralelo al surgimiento de las Ciencias cognitivas. A la Psicología cognitiva también se la ha denominado Psicología de procesamiento de información, e incluso psicología computacional. Ambas expresiones atienden a la tesis básica de que los seres humanos, en especial, al igual que los computadores son sistemas de procesamiento de información. Este es un rasgo básico de la Psicología cognitiva.

Importa ocuparse de si, dado el supuesto básico en el que se basa este enfoque científico, el cerebro es algo más que un dispositivo de procesamiento de información. Desde luego, la analogía cerebro-ordenador se ha demostrado útil para la investigación en lo relativo a cómo se organiza la información y los programas con relativa independencia del soporte físico del sistema, cómo se almacena la información en ciertos dispositivos potenciales de la memoria (como mente inconsciente, en el sentido dado por Jackendoff [10]). Que el hombre-educando es un procesador de símbolos es un presupuesto comúnmente aceptado por los cognitivistas; el problema reside en si el hombre "es algo más que eso, que un procesador de símbolos" [11]. De aquí se deduce la paradoja computacional (de Gardner [12]) según la cual la analogía del ordenador, la teoría de la mente computacional, que nos ha ayudado a simular cómo opera la mente humana, nos permite verificar que el hombre es algo más que eso toda vez que la teoría computacional no resolvería la cuestión de cómo opera el percatamiento consciente y la experiencia propia del mundo.

Volvamos a la revolución cognitiva. Otra característica que la define es la del mentalismo, es decir, su clara oposición al conductismo clásico al postular la idea de que existe, en los seres inteligentes, un sistema de estados internos que explican su conducta. Entre los estímulos y las respuestas existen procesos internos (los auténticos procesos mentales) que son "la causa" 
de su conducta. Tales estados internos son portadores y manipuladores de información, por lo que serían procesos de cognición.

\section{Las teorías de la mente.}

Los especialistas destacan aquí tres teorías o enfoques distintos acerca del funcionamiento de la mente como procesamiento de información, que conviene resumir brevemente: a) aproximación funcionalista de la mente; b) la mente como sistema de representación; c) enfoque de la neurociencia.

El primer enfoque sostiene que cualquier fenómeno psicológico es generado por algún procedimiento efectivo o conjunto de instrucciones que se pueden especificar de manera precisa (algoritmos) y que definen la sucesión de los estados mentales dentro de la mente. Hay que aclarar aquí que tales "algoritmos" no siempre hay que entenderlos en sentido "fuerte" (procedimientos mecánicos sometidos a reglas fijas), sino que con frecuencia son "algoritmos" en sentido "débil" (ordenación racional de pasos, caracterizada por la posibilidad de improvisar o completar algún paso, con lo que cabe un resultado variable dentro de un marco genérico [13]).

La segunda perspectiva defiende que la mente es un sistema de representación y entiende a la psicología como el estudio de los diversos procesos computacionales según los cuales se construyen, organizan, interpretan y se transforman las representaciones mentales. Aquí es usual que los psicólogos cognitivos al hablar de la mente y sus procesos o eventos se refieran a la intencionalidad, a la conciencia o a los estados mentales como estados intencionales. El concepto de "intencionalidad" se usa, en la filosofía medieval, para referirse a fenómenos u operaciones mentales. No se debe confundir con el término más familiar de "intencional" que significa "ánimo", "designio", "con propósito". Así, la intencionalidad se refiere a esa propiedad de "ser acerca de" o de "versar sobre otra cosa", y no necesariamente de hacer algo con un propósito o con intención. De modo genérico, la intencionalidad se puede definir, con Searle como "aquella propiedad de muchos estados y eventos mentales en virtud de la cual éstos se dirigen a, o son sobre o de, objetos y estados de cosas del mundo" (1992: 17). Según esto, siempre que hay intencionalidad -un estado mental intencional- se da una creencia o deseo que puede ser tanto correcto como falso (por ejemplo, una "falsa creencia"). Cuando la mente alberga experiencias que cursan con un percatamiento consciente (sea un percatamiento primario o séalo reflexivo, esto es, ulterior y más elaborado) hablamos, en términos de Jackendoff, de mente fenomenológica.

Por último, según el tercer enfoque los psicólogos se interesan por los tipos de operaciones lógicas o relaciones funcionales que podrían incorporarse en las redes neuronales.

\section{El enfoque neurológico de la acción.}

Por ser la educación una acción, y la Pedagogía una teoría tecnológica sobre la acción, a la Pedagogía cognitiva le interesa la mente en relación con la acción. Un problema fundamental es cómo se configuran las representaciones sobre la acción, cuestión que se basa en la teoría neurológica de la acción.

Una primera observación de los investigadores sobre este campo es que incluso los movimientos más simples del sistema nervioso (los movimientos reflejos a partir de la estimulación cutánea, los reflejos flexores o extensores o de tipo postural, ciertas contracciones musculares elicitadas experimentalmente, etc.) parecen organizarse de una manera esquemática, coordinada y propositiva. Hablar de coordinación significa aquí que estos movimientos se producen conforme a una combinación secuencial, a una secuencia programada u ordenada, y comprometen al conjunto del organismo vital. 
Al considerar estos problemas, la neurociencia de la acción, en correspondencia con las preocupaciones de la psicología cognitiva, somete a investigación el problema de la acción como un problema de coordinación Jeannerod (1997). Importa estudiar aquí como se establecen los modelos internos acerca de la acción y cómo ciertos organismos inteligentes, no sólo reaccionan a las perturbaciones del entorno, sino que también inician o emprenden las acciones. Se forman representaciones internas acerca de cómo es el mundo exterior, anticipan la representación de cómo quedaría modificado ese mundo a partir de una determinada acción sobre el mismo, y actúan consecuentemente. Este proceso refleja de forma bastante aproximada cómo se produce la acción humana sobre el entorno (por ejemplo, en una acción de intervención pedagógica).

En este proceso, es importante cómo se registran y recuperan las informaciones internas acerca de los patrones de referencia (de riesgo tolerable, de disonancia admisible, de seguridad, de calidad mínima u óptima, etc.). Se implican aquí conceptos de asentada tradición en la biología y en la psicología cognitiva, tales como los de esquema, de autorregulación y de engrama. La pedagogía se habrá de preocupar por la generación de los esquemas anticipatorios sobre la acción, esquemas que actúan, no sólo cognitiva, sino metacognitivamente, archivando y operando sobre informaciones, tanto de su entorno, como de su propios mecanismos cognitivos (Jeannerod: 1997, 5). El desarrollo de la planificación de una tarea puede verificarse de dos formas cualitativamente diferentes: ya mediante la selección y aplicación de rutinas bien definidas, ya a través de una serie de pasos para especificar la meta, configurar un plan y definir las posibles contingencias temporales (Jeannerod, 1997: 127).

\section{5. Ámbito de las Ciencias cognitivas.}

En un sentido amplio suele usarse esta expresión para indicar o hacer notar que el estudio de la mente humana es en sí mismo una empresa científicamente válida y relevante. Pero las Ciencias cognitivas no constituyen un campo disciplinar nítidamente delimitado. Más bien se trata de un campo de investigación y de estudio interdisciplinar.

Acostumbra a decirse que la Inteligencia Artificial ocupa un puesto de privilegio dentro de Ciencias cognitivas y que el resto de las disciplinas son: lingüística, neurociencia, psicología y, también, la antropología y la filosofía [14]. De modo específico, según la exposición de Martínez Freire (1995) dentro del ámbito de las Ciencias cognitivas, podrían distinguirse tres campos disciplinares más específicos: a) ciencias básicas: psicología cognitiva e inteligencia artificial; b) ciencias instrumentales: lógica, informática, neurociencia y lingüística; y c) metaciencia cognitiva: filosofía de la mente.

La evolución del campo interdisciplinar de las Ciencias cognitivas pone de manifiesto, como dicen Varela y otros (1992) que existe una "historia humana" de las ideas acerca del autoconocimiento humano y del conocimiento de la mente y de la cognición. En esta historia o mutación, el conocimiento se ha ligado de forma tangible y muy estrecha con una tecnología que transforma las prácticas sociales que, a su vez, dieron origen a la misma tecnología. La inteligencia artificial es un ejemplo visible de ello.

Según estos autores, la tecnología actúa como amplificador, en el sentido de que no se pueden separar las Ciencias cognitivas de las "tecnologías cognitivas" sin despojar a ambas de su elemento complementario. Es decir, a través de la tecnología el estudio científico de la mente proporciona a la sociedad un espejo que transciende un sólo círculo disciplinar. Por esto mismo las Ciencias cognitivas no constituyen un campo monolítico y único de investigación, aunque hay polos de dominación en su historia y evolución como área de estudio. 
Se pueden así distinguir tres etapas sucesivas en las ciencias cognitivas:

A) Cognitivismo ("paradigma simbólico" o "enfoque informático”). Se apoya en la hipótesis de que la cognición es la manipulación de símbolos, al estilo de lo que hacen los ordenadores digitales. La cognición es la representación mental: se piensa que la mente opera manipulando símbolos que representan rasgos del mundo como si éste fuera de tal manera. De acuerdo con ello, el estudio de la cognición, en tanto que representación mental, proporciona un dominio autónomo de estudio para las C.C. que, se supone, es independiente de la neurobiología, por un lado, y de la sociología y de la antropología, por otro.

B) Emergencia. Esta perspectiva surge como crítica a la idea del procesamiento de símbolos como vehículo apropiado para las representaciones. También se le denomina "conexionismo", término que deriva de la idea de que muchas tareas cognitivas (visión, memoria, etc.) parecen manipularse mejor mediante sistemas integrados por muchos componentes que, cuando se conectan mediante las reglas apropiadas. Para este enfoque, una representación consiste en una correspondencia entre un estado global emergente y las propiedades del mundo. No es una función de símbolos particulares (por ejemplo, el "emergentismo" de Searle).

C) Enacción. Este enfoque pone en cuestión el concepto de representación como concepto central de las ciencias cognitivas. La idea de la cognición como representación, según esta perspectiva, oculta tres supuestos fundamentales: $1^{\circ}$ ) que habitamos un mundo con propiedades particulares (longitud, color, sonido, movimiento, etc.); $2^{\circ}$ ) que captamos $o$ recobramos estas propiedades representándolas internamente; $3^{\circ}$ ) que un "nosotros" subjetivo separado es quien hace estas cosas. Estos tres supuestos implican un fuerte compromiso, tácito e incuestionado, con el realismo o el objetivismo/subjetivismo acerca de cómo es el mundo, qué somos nosotros y cómo llegamos a conocer el mundo. Para la "enacción", la cognición no es la representación de un mundo "pre-dado" por una mente "pre-dada", sino más bien la puesta en obra de un mundo y una mente a partir de una historia de la variedad de acciones que un ser realiza en el mundo. La mente no es, como reza el conocido título del libro de Rorty (La filosofía y el espejo de la naturaleza), el "espejo de la naturaleza”.

\section{Los problemas mente-mente y mente-cuerpo.}

Un problema teórico que, desde el punto de vista de la filosofía de la mente, es fundamental en este campo de estudio es el clásico problema de la relación mente-cuerpo. Pero, junto a éste, y todavía más básico que él, nos encontramos con el problema mente-mente.

Éste ultimo problema puede considerarse como el problema más básico. De nuevo nos remite a la cuestión de la suficiencia-insuficiencia de la teoría computacional de la mente. Volviendo al pensamiento de Jackendoff, no podemos reducir la conciencia a la mera autorreferencia toda vez que ésta incluye, también, la autoconciencia y, en última instancia, la conciencia de la experiencia de las cosas. Si, por un momento, vamos más allá de lo que Searle entiende por actos intencionales, no puede admitirse que ningún ordenador pueda experimentar el mundo. Nos encontramos en un punto crucial de las teorías sobre la mente y para una antropología pedagógica de cariz cognitivo. Cuando autores como Putnam creen ver en las teorías computacionales la solución a la relación mente-cuerpo lo que están aportando es una aproximación (Jackendoff, 1998: 39) al problema mente computacional-cuerpo olvidando plenamente el problema mente fenomenológica-cuerpo. Pero también es cierto que profundizar en cómo opera la mente computacional ayuda a comprender mejor la mente fenomenológica.

Por lo demás, el problema derivado mente-cuerpo se puede plantear así: ¿Cómo es posible 
que una realidad física y tangible como el cerebro pueda dar lugar como resultado a realidades intangibles y no físicas como los pensamientos y los procesos mentales que los causan? Los procesos mentales, ¿son procesos físicos cerebrales, de carácter neurofisiológico, o emergen de alguna entidad no física, más o menos misteriosa y desconocida?

Como se sabe, Descartes estableció una distinción entre pensamiento (mente, res cogitans) y cuerpo (res extensa) y sostuvo que el pensamiento carecía de extensión. Este planteamiento implica la introducción de un dualismo en el estudio de la mente y conduce al problema de la explicación psicológica, es decir, a la posibilidad de una explicación de los procesos mentales que debe ser distinta de la explicación puramente física. Por otra parte, plantea el problema mente-cuerpo como un problema real, es decir, como un problema posible a partir, precisamente, del anterior dualismo cartesiano.

Según Searle (1996), éste es un problema central en la filosofía de la mente contemporánea, problema que, a su juicio, puede tener una solución teórica relativamente fácil, pero que muchos estudiosos de este campo se niegan a reconocer o a aceptar. Para este autor la solución, que diversas teorías acerca de la mente parecen rechazar, sería la siguiente: los fenómenos mentales están causados por procesos neuropsicológicos del cerebro y son a su vez rasgos del cerebro (naturalismo biológico). O, dicho de otro modo, quizá más claro aún: todos nosotros tenemos estados de conciencia cualitativamente subjetivos, y tenemos estados mentales intrínsecamente intencionales, tales como creencias, deseos, intenciones y percepciones. Y tanto la conciencia como la intencionalidad son procesos biológicos causados por procesos neuronales que "tienen lugar", que acontecen en el cerebro. Pero ni la conciencia (de nuevo el problema de la conciencia), ni la intencionalidad se podrían reducir, según esta posición, a algo distinto de ellas mismas.

De acuerdo con este enfoque, mente y cuerpo interactúan, aunque no son dos entidades enteramente diferentes, ya que los fenómenos mentales son rasgos del cerebro. Es decir, podemos defender simultáneamente que los procesos mentales no son independientes de los procesos físicos (de los procesos cerebrales, en concreto) y que los procesos mentales no se reducen a meros procesos cerebrales, tal y como los estudian en la neurociencia. Esta posición puede denominarse emergentismo: la teoría que afirma que los procesos mentales aparecen como fenómenos o propiedades que "emergen" de los procesos cerebrales. Las propiedades mentales son propiedades emergentes de los sistemas neurofisiológicos, pero no de algún proceso no físico de carácter misterioso.

Esta tesis se niega hoy desde diversos puntos de vista dominantes en el panorama contemporáneo de la filosofía de la mente, como el enfoque materialista, frente al cual se opone el enfoque del dualismo de propiedades, que en sustancia acepta el legado de Descartes. Según Searle, no hay por qué elegir entre ambos enfoques. Se pueden aceptar los hechos obvios de la física -por ejemplo que el mundo consta de partículas de física en campos de fuerza- sin negar por ello que entre los rasgos físicos del mundo haya o se dan fenómenos biológicos tales como estados de conciencia cualitativamente internos e intencionalidad intrínseca. Para otros, la mente computacional nunca podrá adquirir conciencia de sí y menos conciencia de la experiencia significativa del mundo de la vida.

Conforme con una interpretación materialista, los fenómenos mentales causados por procesos neuropsicológicos del cerebro, como las intenciones, los deseos, las creencias, la conciencia, etc., son reducibles a fenómenos puramente físicos del cerebro. Lo que hace este enfoque es "naturalizar" los fenómenos mentales, la intencionalidad o los estados mentales intencionales.

Este enfoque se articula en forma de seis teorías inverosímiles, como las llama Searle, de la mente humana. Las teorías son las siguientes:

$1^{\mathrm{a}}$. Los estados mentales, como tales, no existen (materialistas eliminativos). 
$2^{\mathrm{a}}$. El enfoque psicológico que da cobertura a la afirmación de la existencia de estados mentales intencionales (psicología popular) es simplista y falsa.

$3^{\text {a }}$. No hay nada específicamente mental en los llamados estados mentales, sino que son relaciones causales entre sí y con los inputs y outputs del sistema de cual forman parte (funcionalismo).

$4^{\mathrm{a}}$. Los ordenadores pueden pensar inteligentemente, pueden tener pensamientos, así como sentimientos y comprensión, en virtud de la mejora del programa que se le aplique (inteligencia artificial en su acepción más fuerte).

$5^{\text {a }}$. Términos de nuestro vocabulario mental como creencia, deseo, temor, etc., no son más que un modo de hablar, un vocabulario útil para explicar la conducta, pero no términos que indican la existencia de estados mentales o psicológicos subjetivos.

$6^{\mathrm{a}}$. La conciencia, entendida como fenómenos cualitativos de sentir o darse cuenta de forma privada o subjetiva, no existe en absoluto [15]. Queda negada así la introspección y la conciencia de sí mismo como mí mismo.

Lo que hacen estas teorías "materialistas" sobre la mente es negar lo que para Searle son los cuatro rasgos esenciales de los fenómenos mentales, rasgos que, según esta forma de pensar, no serían difíciles de encajar en una visión del mundo como compuesto solamente de cosas o propiedades materiales:

1. Conciencia. En el sentido de "darnos cuenta" de lo que hacemos, es decir, como una forma de "atención".

2. Intencionalidad. Rasgo mediante el cual nuestros estados mentales se dirigen o se refieren a objetos o estados de cosas del mundo distintos de los propios estados mentales (deseos, esperanzas, temores, creencias, intenciones, etc. tienen intencionalidad en cuanto se dirigen a un mundo distinto de la mente).

3. Subjetividad de los estados mentales. La subjetividad indica el hecho de que cada uno puede conocer sus estados mentales internos mientras que los demás no pueden conocerlos de forma directa. Es lo que se llama el privilegio del acceso directo de cada sujeto humano a sus propios procesos mentales.

4. Causación mental. Nuestros pensamientos y sensaciones tienen de hecho algún efecto causal sobre el mundo físico (decido levantar mi brazo y mi brazo se levanta). Los procesos mentales causan efectos físicos.

Siguiendo esta vía, podríamos concluir, por lo tanto, que los cerebros causan las mentes. Pero los procesos mentales no existen al margen de los procesos cerebrales neuronales, aunque son procesos diferenciados de ellos, lo mismo que las propiedades superficiales de una mesa (como su solidez), que se explica por la estructura de enrejado ocupada por las moléculas de las que se compone, se diferencia de estos microelementos. Es posible, por tanto, distinguir el estudio de los procesos mentales, tal y como los estudia la psicología de los procesos cerebrales tal y como los estudia la neurociencia.

El aspecto más positivo de la teoría de Searle es que sus explicaciones ayudan a no sucumbir en un reduccionismo (los procesos mentales causados por procesos cerebrales no se reducen a procesos físicos neuronales), con lo cual la psicología mantiene su autonomía frente a la neurociencia, a la que no se reduce en su estudio de la mente, pero en cuyos resultados se apoya. Su aspecto más negativo es que al hacer depender los procesos mentales de procesos cerebrales, excluye la posibilidad de la existencia de mentes mecánicas o de mentes no 
naturales, frente a la evidencia de la Inteligencia Artificial y de máquinas que piensan. El biologismo de Searle le impide aceptar el hecho de que los procesos mentales pueden tener una base física distinta de los sistemas neuronales.

\section{7. ¿El hombre, algo más hábil resolutor de problemas?}

Para algunos autores, el enfoque emergentista, lo mismo que el cognitivismo, como núcleo central de las ciencias cognitivas reducen la cognición a la mera resolución de problemas. En ambos casos se comparte un cierto "realismo cognitivista", que es el supuesto según el cual el mundo se puede dividir en regiones de elementos y tareas y que la cognición cosiste en resolver problemas que, para que tengan éxito, deben respetar los elementos, las propiedades y las relaciones de estas regiones pre-dadas.

Este enfoque mentalista dominante, que pone el acento en el procesamiento de información, funciona hasta cierto punto en tareas donde resulta fácil especificar todos los elementos posibles. Pero hay tareas que exigen, además, otros elementos menos especificables a priori, como, por ejemplo, las habilidades motrices adquiridas, el uso continuo del sentido común, el conocimiento práctico, etc.

Así, las teorías de la enacción acentúan el hecho de que el conocimiento es el resultado de una interpretación que emerge de nuestra capacidad de comprensión, la cual está arraigada en la estructura de nuestra corporalidad (en nuestra corporización) se vive o experimenta dentro de un dominio de acción consensual y de un contexto cultural. El mundo, nuestro mundo, adquiere sentido en la medida que nos percibimos como seres activos que actúan en él negociando el significado de las acciones con otros seres activos dentro de un marco cultural. Se puede decir, así, que la cultura forma y conforma la mente humana. Esta es la perspectiva defendida por Bruner, para quien, como vimos, la revolución cognitiva operada en psicología se alejó de sus propósitos iniciales, que él centra en la "búsqueda del significado".

Frente a la perspectiva del enfoque computacional, Bruner, junto a muchos otros, defiende otra de carácter psicocultural, que se dirige sobre todo a los procesos de interpretación y elaboración de sentido. La primera perspectiva se interesa sobre todo en el procesamiento de la información, es decir el cómo la información -finita, codificada y precisa- es organizada por un mecanismo computacional.

Aquí la información se toma como un material dado y ya establecido en relación con algún código preexistente y regulado por reglas. La información comprende un mensaje que ya ha sido previamente codificado en el sistema. El significado se asigna a los mensajes del ordenador, y es grabado en su sistema, pero no es un descubrimiento resultante del proceso mismo de computación. Así, es sistema es "ciego" ante el hecho de si lo que incluye son poemas, cifras o lo que sea. De acuerdo con esto, un mensaje es informativo si reduce al mínimo posible el número de elecciones alternativas, lo que implica la existencia de un código de elecciones posibles establecidas previamente. Un sistema de este orden nada puede hacer frente a la vaguedad, la polisemia o las conexiones metafóricas. El procesamiento de información tiene necesidad, para que funcione con éxito, de planificación previa y reglas precisas.

Un enfoque más adecuado, y pedagógicamente relevante según Bruner, para el estudio de la mente es el que pone el énfasis en la relación entre mente y cultura. La mente es, de acuerdo con este punto de vista "superorgánica" y da forma o conforma la mente del individuo. Su expresión es la creación de significados, es decir, la atribución o asignación de significados a cosas en diferentes contextos. Esto supone situar los encuentros con el mundo en contextos culturales concretos. Es este carácter situado de los significados lo que asegura su comunicabilidad y negociabilidad. 
Existen diversas razones para reivindicar el importante papel de la cultura en la conformación de la mente:

a) en primer lugar, los seres humanos no terminan en su propia piel, sino que son expresión de la cultura. No existe una naturaleza humana independientemente de la cultura, y el hecho de la participación del hombre en la cultura hace inviable construir una psicología humana basada sólo en el individuo concreto;

b) en virtud de nuestra participación en la cultura, el significado se hace público y compartido. El significado se negocia y nos hace interactuar con otros en contextos culturales públicos, intersubjetivos;

c) por último, el hecho de la interacción con otros, la práctica de la intersubjetividad, hace que la psicología popular sea un buen marco teórico para revitalizar la importancia de los estados intencionales, los deseos, creencias e intenciones.

Así, pues, de acuerdo con esta perspectiva, la mente no funciona sólo procesando información, sino que su funcionamiento humano apunta a la creación, comunicación y negociación de significados.

\section{Consecuencias para la investigación y la práctica educativas.}

Los tres puntos clave que se han destacado aquí son: el papel de la educación (y, de forma implicada, de la inteligencia) en la sociedad del conocimiento, el estudio de la mente y de los tipos de mente y la teoría neurológica de la acción. Los tres constituyen los núcleos más significativos de una pedagogía cognitiva concebida como disciplina académica y como programa interdisciplinar de investigación.

El tratamiento de estos problemas permitirá en el futuro, quizá, establecer un espacio para la Pedagogía en su devenir desde una pedagogía científica a una tecnológica, a una tecnológicocognitiva y a una cognitivo-cultural (o, simplemente, cognitiva que atiende, tanto a la mente fenomenológica como a la computacional, y tanto a la cognición como al pensamiento).

Los campos específicos en los que se puede emplear este enfoque pedagógico son todos aquellos en los que opera la pedagogía sintética: la pedagogía informática, el cognitivismo sistémico aplicado a los problemas culturales (interculturales) y medioambientales o a cualquier problema que haya de examinarse desde la perspectiva de la globalización y la interciencia, la formación del pensamiento de los profesionales de la educación, la pedagogía de la ciencia, etc.

La experiencia académica permite asegurar que esta disciplina y este programa de investigación permite acondicionar bien la mente de los estudiantes de pedagogía en su último año de estudios.

\section{BIBLIOGRAFÍA}

Bruner, J. (1991) El estudio apropiado del hombre, p. 19-46 en Actos de significado. Más allá de la revolución cognitiva. Madrid: Alianza.

Bruner, J. (1997) Cultura, mente y educación, p. 19-62 en La educación, puerta de la cultura. Madrid: Visor.

Gardner, H. (1987)The mind's new science. A history of Cognitive Revolution. N. York: Basic Books.

Jackendoff, R. (1998) La conciencia y la mente computacional. Madrid: Visor. 
Jeannerod, M. (1997) The cognitive neuroscience of action. London: Blackwell.

Martí, E. (1997) Estados mentales, p. 15-24 en Construir una mente. Barcelona: Paidós.

Martínez-Freire, P. (1995) Mentalismo y psicología cognitiva, p. 143-154 en La nueva filosofía de la mente. Barcelona: Gedisa.

Searle, J. R. (1992) La naturaleza de los estados intencionales, p. 17-50 en Intencionalidad. Un ensayo en la filosofía de la mente. Madrid: Tecnos.

Searle, J. R. (1996) ¿Qué marcha mal en la filosofía de la mente?, p. 15-40 en El redescubrimiento de la mente. Barcelona: Crítica.

Varela, F.J. y otros (1992) Una circularidad fundamental: en la mente del científico reflexivo, p. 27-38 en De cuerpo presente. Las ciencias cognitivas y la experiencia humana. Barcelona: Gedisa.

Vázquez, G. (1991) La Pedagogía como ciencia cognitiva, p. 123-146 en Revista Española de Pedagogía 49 (188).

\section{NOTAS}

[1] G. Vázquez (1991) La Pedagogía como ciencia cognitiva, p. 123 en Revista Española de Pedagogía 49 (188).

[2] A este enfoque responde la obra de J.-P. Astolfi, A. Giodan, G. Aohau, V. Host, J-L. Martinand, G. Rumelhard y G. Zadounaïsky (1978) Quelle éducation scientifique pour quelle societé. París: P.U.F. Ver, sobre todo, la segunda parte.

[3] Es la pregunta de W. Heisenberg (1974) Más allá de la física. Atravesando fronteras. Madrid: B.A.C, p. 221-232. Confiesa el autor que en un principio estuvo tentado de titular a ese capítulo "¿Cómo se hace una revolución?”; nosotros habríamos de preguntarnos si estamos en condiciones de hacer una revolución pedagógica, queremos decir la necesaria dimensión pedagógica de la ya producida revolución científica.

[4] Así lo entiende J. Ma Asensio (1997) Biología y educación. El ser educable. Barcelona: Ariel; cap. $2^{\circ}$

[5] F. Sáez Vacas (1987) Computadores personales. Hacia un mundo de máquinas informáticas. Madrid: Fundesco.

[6] Luhman propone que "los sistemas comprenden a los sistemas". N. Luhmann (1992) Teoría de la sociedad y pedagogía. Barcelona: Paidós.

[7] En este sentido, las antiguas empresas industriales están evolucionando hacia el modelo de empresas "cuaternarias" concebidas como organizaciones de conocimiento cooperativo y reticular. Esto debería aplicarse a toda organización educativa y, desde luego, a la universidad.

[8] H. Arendt (1996) La condición humana. Barcelona: Paidós, 187-188.

[9] Estas preguntas no tienen por qué ser necesariamente disyuntivas. Cabe la posibilidad de explicar y comprender, al mismo tiempo, al hombre como artífice y artefacto, tal como sugiere Bolter. Ver: Sáez Vacas. Ordenadores personales, p. 162.

[10] R. Jackendoff (1998) La conciencia y la mente computacional. Madrid: Visor, cap. 2.

[11] Vázquez (1991) La Pedagogía como ciencia cognitiva, p. 13.

[12] H. Gardner (1987) The mind's new science. A history of Cognitive Revolution. N. York: 
Basic Books.

[13] Queda implicada aquí cómo procesan la información la mente de los expertos. Ver el trabajo clásico de H. L. Dreyfus y S. E. Dreyfus (1986) Mind over machine. N. York: Free Press.

[14] No se incluye, como se ve, la pedagogía; no obstante, en un trabajo tan significativo como el de A. Collins (1977) Why cognitive Science. Cognitive Science I (1), p.1, se incluye la tecnología de la educación. En estos veinte años son numerosos los trabajos pedagógicos aceptados en las revistas de ciencia cognitiva. En España son, hasta ahora, muy contados, pudiéndose citar el enfoque cognitivo que se da al conocimiento pedagógico por A. García del Dujo y J. García Carrasco (1996) Teoría de la educación. I. Educación y acción pedagógica. Salamanca: Universidad de Salamanca. En trabajos más específicos debe señalarse los de A. J. Colom (1995) De la educación ambiental al cognitivismo sistémico, p. 49-58 en P. Ortega y F. López (coords.) Educación ambiental: cuestiones y propuestas. Murcia: Cajamurcia; y A. J. Colom (1997) La regionalización de la educación como tecnología cognitiva virtual. Teoría de la Educación 9, 7-19.

[15] Se produce en este punto con la cuarta objeción acerca de si pueden pensar las máquinas según A. M. Turing (1974) ¿Puede pensar una máquina? Universidad de Valencia: Dept ${ }^{\circ}$ de Lógica y Filosofía de la ciencia, p. 39.

(C) Ediciones Universidad de Salamanca. 